\title{
LA FUNCIÓN DEL JUEGO EN EL DESARROLLO INTEGRAL DE LOS NIÑOS DE 2 A 4 AÑOS. ESTUDIO DESDE LA PERSPECTIVA DE LOS PADRES DEL CENTRO INFANTIL DEL BUEN VIVIR TIERRA NUEVA
}

\section{THE ROLE OF PLAY IN THE INTEGRAL} DEVELOPMENT OF CHILDREN FROM 2 TO 4 YEARS. STUDY FROM THE PERSPECTIVE OF THE PARENTS OF THE CHILDREN'S CENTER OF BUEN VIVIR TIERRA NUEVA

\author{
SUSANA BALDEÓN LOZA ${ }^{1}$ \\ MAYRA VELÁSTEGUI VILLAMARÍN ${ }^{2}$ \\ ESTEFANY HEREDIA MENA ${ }^{3}$
}

\footnotetext{
${ }^{1}$ Universidad Central del Ecuador, Facultad de Ciencias Psicológicas, Quito, Ecuador (ssbaldeon@uce.edu.ec).

${ }^{2}$ Pontificia Universidad Católica del Ecuador, Facultad de Psicología, Quito, Ecuador (mvelastegui946@puce.edu.ec)

${ }^{3}$ Universidad Central del Ecuador, Facultad de Ciencias Psicológicas, Quito, Ecuador (psi.herediamena@gmail.com)
} 



\section{LA FUNCIÓN DEL JUEGO EN EL DESARROLLO INTEGRAL DE LOS \\ NIÑOS DE 2 A 4 AÑOS. ESTUDIO \\ DESDE LA PERSPECTIVA DE LOS PADRES DEL CENTRO INFANTIL DEL BUEN VIVIR TIERRA NUEVA}

\section{THE ROLE OF PLAY IN THE INTEGRAL DEVELOPMENT OF CHILDREN FROM 2 TO 4 YEARS. STUDY FROM THE PERSPECTIVE OF THE PARENTS OF THE CHILDREN'S CENTER OF BUEN VIVIR TIERRA NUEVA}

Susana Baldeón, Mayra Velástegui, Estefany Heredia

Palabras clave: Función del juego, niños en edad preescolar, creencias parentales, valoración del juego

Key words: Role of play, Children in preschool, Parental beliefs, Assessment of the game.

\section{RESUMEN}

Este artículo plantea como eje de investigación al juego en el desarrollo infantil. El objetivo es valorar el papel que los padres, de los niños y niñas de 2 a 4 años del Centro Infantil del Buen Vivir "Tierra Nueva", le atribuyen al juego en el desarrollo físico, cognitivo, socioemocional y del lenguaje. El estudio se encuen- 
tra sustentado en las teorías socio-históricas y cognitiva del juego. Es una investigación de tipo descriptiva, diseño no experimental, enfoque cuantitativo, realizado en una muestra de 50 padres, los datos se obtuvieron mediante una encuesta y la entrevista clínica. Se concluye que la mayor parte de los padres (58\%) considera importante la función general del juego en el desarrollo infantil, sin embargo, se deprecia su papel en la estimulación del área cognitiva en el $68 \%$ y del lenguaje en el $56 \%$, evidenciando frecuencias de juego bajas entre padres e hijos en estas áreas. Se recomienda la estructuración y aplicación de un programa que incluya capacitación y talleres que procuren la concienciación en los padres sobre la importancia del juego y su función en todas las áreas del desarrollo y el rol parental en las conductas lúdicas infantiles.

\section{ABSTRACT}

This article raises as a research axis the role of plan in the development of children. The objective is to assess the role that parents of children from 2 to 4 years of the Buen Vivir Children's Center "New Earth" attribute to play in physical, cognitive, socio-emotional and language development. The study is based on the socio-historical and cognitive theories of the game. It is a research of descriptive type, non-experimental design, quantitative approach, performed in a sample of 50 parents, data were obtained through a survey and clinical inter- view. It is concluded that most of the parents (58\%) consider the general role of play in child development to be important, however, their role in the stimulation of cognitive area in 68\% and language in $56 \%$ is depreciated, Evidencing low frequencies of play between parents and children in these areas. It is recommended the structuring and implementation of a program that includes training and workshops to raise awareness in parents about the importance of play and its role in all areas of development and parental role in children's play behavior.

\section{INTRODUCCIÓN}

La constitución del Ecuador niñas, niños y adolescentes tendrán de(2008) en su artículo 44 menciona: "Las recho a su desarrollo integral, entendido 
como proceso de crecimiento, maduración y despliegue de su intelecto y de sus capacidades, potencialidades y aspiraciones" ( $p$.34).

En este marco, de los doce objetivos del Plan Nacional del Buen Vivir 2013-2017, el objetivo número dos propone: Auspiciar la igualdad, la cohesión, la inclusión y la equidad social y territorial en la diversidad; se incluye como política "garantizar el desarrollo integral de la primera infancia, a niños y niñas menores de 5 años". (SENPLADES, 2013, pág. 127)

El desarrollo infantil se enmarca como un proceso multidimensional en el que Feldman (2008) lo precisa como el estudio de los patrones de crecimiento, cambio y estabilidad que ocurren desde la concepción, influenciados por factores sociales como las limitaciones económicas o problemáticas familiares, mientras que la Organización Mundial de la Salud (2013) destaca la autonomía en la que "... los niños progresan desde un estado de dependencia de todos sus cuidadores en todas sus áreas de funcionamiento, durante la lactancia, hacia una creciente independencia en la segunda infancia, la adolescencia y la adultez. (p.7)

La etapa preescolar de niños entre dos y seis años, se caracteriza por un mayor dominio de habilidades neuromusculares, el inicio de la socialización, logro de autonomía temprana y el pensamiento simbólico. Hoy en día, los estu- dios revelan que muchos de los desafíos afrontados por la población adulta relacionados con problemas de salud mental, obesidad, retardo en el desarrollo, enfermedades cardíacas, criminalidad, habilidad numérica y de lecto-escritura, entre otros, tienen sus raíces en la etapa preescolar (Irwin, 2007).

Hoffman, Paris y Hall (1995) describen al desarrollo como un proceso acumulativo, holístico y con direccionalidad, donde las experiencias previas en situaciones semejantes influencian los aprendizajes posteriores; de manera que las adquisiciones más simples de los primeros estadios, son la base para otros más complejos.

Dentro de estos factores la relación de padres e hijos es fundamental. "La familia juega un papel crucial en el desarrollo de los niños y niñas, tanto que se puede afirmar que es el contexto de desarrollo por excelencia durante los primeros años de vida de los seres humanos" (Muñoz, 2006, pág. 1). Justificado en la cercanía, que la familia mantiene con el infante y la intensidad de las relaciones que entablan. Es el primer referente del mundo que el niño adquiere.

Los padres desempeñan los roles de socialización, educación, protección, cuidado y estimulación. En este último se busca potencializar las áreas del desarrollo en los infantes: motora, cognitiva, socio-afectiva y del lenguaje. "Es aportar 
a los hijos estimulación que garantice que se pueden desarrollar correctamente en su entorno físico y social" (Salles, 2011, pág. 30).

Dentro de este rol, se encuentra la participación de los padres en el juego infantil, pues este es el principal medio de estimulación de los niños, sobre todo en la etapa preescolar. Los padres son los promotores del juego en sus distintos niveles de ejercicio, simbólico o reglado.

El estudio propuso describir la función del juego desde la percepción de los padres de los niños y niñas de 2 a 4 años del C.I.B.V. "Tierra Nueva".

Se espera conocer las funciones que los padres le atribuyen al juego, considerando que son estos quienes estimulan o no el juego infantil. Muchos de los padres y madres de familia, pueden considerar el juego, como una actividad de distracción y poco compromiso, sin embargo que el juego implica una serie de procesos que contribuyen al crecimiento integral del infante (Arango, 1997).

Conocemos la valoración que los padres han adjudicado al juego, respecto a su función, se pueden establecer objetivos dentro de la estimulación infantil y proyectos direccionados hacia los padres y su conocimiento sobre el desarrollo de sus hijos.

A partir de los resultados obtenidos en la investigación se busca concienciar a las instituciones, los padres y academia sobre la importancia que las creencias y actitudes parentales respecto al juego tienen en el desarrollo preescolar, para de esta manera, fomentar la corresponsabilidad de los padres en la estimulación de los niños, que hasta estos momentos ha sido principalmente relegada a los Centros de Desarrollo Infantil e instituciones educativas representadas en esta investigación en el centro infantil del buen vivir. 


\section{MATERIAL Y MÉTODOS}

La presente investigación fue de tipo descriptiva, de diseño no experimental, con un enfoque cuantitativo, llevada a cabo a partir de la construcción y validación de un cuestionario, sumado a las entrevistas y estructuración de historias clínicas.

La población se constituyó a partir de 105 padres de los niños del C.I.B.V. "Tierra Nueva", que pertenecen al sector sur de la ciudad de Quito principalmente a los barrios Quito Sur y Ajaví, aledaños al centro infantil. Poseen una condición socioeconómica media y baja. La muestra estuvo integrada por 50 padres del centro infantil.

La elección de muestra se realizó a partir de un diseño no probabilístico, se tomaron como criterios de inclusión, a los padres de los niños entre dos y cuatro años, correspondiente a la etapa preescolar. Período caracterizado por un mayor dominio de habilidades neuromusculares, el inicio de la socialización, logro de autonomía temprana y el pensamiento simbólico.
Para la construcción y validación del cuestionario se siguió el procedimiento determinado por Hernández (2010). En primera instancia se realizó la revisión de literatura sobre el objetivo a cumplir, para posteriormente efectuar una definición de las variables a medir y sus indicadores.

El cuestionario se estructuró con treinta preguntas cerradas, mediante el formato de Likert, agrupadas en tres secciones. La primera involucra preguntas de contextualización, la segunda sección presenta preguntas en torno a la valoración de la función del juego y en tercer lugar en relación con la frecuencia de juego en cada función.

La forma de aplicación fue directa, en su mayoría dentro de las sesiones ordinarias de padres. De forma que las respuestas obtenidas no pudieran estar sesgadas por el miedo a ser juzgados por parte del encuestador. Para su calificación se realizó un promedio entre las preguntas que conformaron cada área, descritas en el siguiente apartado. 


\section{Tabla 1. Desarrollo de ítems del Cuestionario por objetivos}

\begin{tabular}{|c|c|c|c|c|}
\hline Variable & Dimensión & Indicador & Subindicador & Items \\
\hline \multirow[t]{6}{*}{$\begin{array}{l}\text { Función } \\
\text { del } \\
\text { juego }\end{array}$} & Física & $\begin{array}{l}\text { Coordinación dinámica } \\
\text { general } \\
\text { Coordinación óculo-ma- } \\
\text { nual } \\
\text { Equilibrio }\end{array}$ & $\begin{array}{l}\text { Marchar, correr, trepar } \\
\text { Recepción, lanzamientos y manipu- } \\
\text { lación de elementos pequeños } \\
\text { Mantenerse sobre un pie, saltar en un } \\
\text { pie, caminar sobre una barra }\end{array}$ & $5,6,7,18,19,20$ \\
\hline & Cognitivo & $\begin{array}{l}\text { Imaginación } \\
\text { Resolución de conflictos } \\
\text { Nociones comparativas }\end{array}$ & $\begin{array}{l}\text { Crea historias, personajes y esce- } \\
\text { narios } \\
\text { Busca opciones, escucha al otro } \\
\text { Clasificación de objetos por tamaño, } \\
\text { color y forma }\end{array}$ & $8,9,10,21,22,23$ \\
\hline & Socioemocional & $\begin{array}{l}\text { Comprensión de reglas } \\
\text { Acciones cooperativas } \\
\text { Expresión de emociones } \\
\text { Sociabilización }\end{array}$ & $\begin{array}{l}\text { Establecen reglas entre todos, respe- } \\
\text { tan las reglas durante el juego } \\
\text { Ayuda al otro, se preocupa por el otro } \\
\text { Entiende sus emociones, Diferencia } \\
\text { sus emociones, puede expresar sus } \\
\text { emociones } \\
\text { Se relaciona con sus pares, aprende } \\
\text { convenciones sociales }\end{array}$ & $11,12,13,14,24,25,26,27$ \\
\hline & Lenguaje & $\begin{array}{l}\text { Adquisición del lenguaje } \\
\text { La asociación del signo al } \\
\text { significante } \\
\text { Aumento de vocabulario }\end{array}$ & $\begin{array}{l}\text { Realiza sonidos durante el juego, } \\
\text { imita sonidos durante el juego } \\
\text { Nombra a los objetos. } \\
\text { Uso de expresiones propias del juego, } \\
\text { aprende el vocabulario de los otros } \\
\text { jugadores. }\end{array}$ & $15,16,17,28,29,30$ \\
\hline & $\begin{array}{l}\text { Frecuencia de } \\
\text { juego }\end{array}$ & Minutos de juego & $\begin{array}{l}\text { Minutos de juego del niño por día } \\
\text { en casa } \\
\text { Minutos de juego de los padres junto } \\
\text { al niño }\end{array}$ & $1,2,3$ \\
\hline & $\begin{array}{l}\text { Percepción } \\
\text { global }\end{array}$ & Conceptualización & $\begin{array}{l}\text { Función general del juego en el } \\
\text { desarrollo infantil }\end{array}$ & Preguntas de la 5 a la 30 \\
\hline
\end{tabular}


Para determinar su validez se utilizó la técnica de juicio de expertos. Dentro de su valoración el experto calificó individualmente cada pregunta bajo tres criterios: congruencia, claridad en la redacción y tendenciosidad en la formulación. Con los puntajes obtenidos se procedió a realizar el cálculo del índice de validez del contenido (IVC) equivalente a 0,986 .

Para determinar el grado de confiabilidad se utilizó el Alpha de Cronbach. En primer término se seleccionó una po- blación de diez padres con características semejantes a la del estudio, donde se aplicó la prueba piloto. La administración fue de tipo directa, de forma que se pudiera determinar las dudas de los participantes sobre el instrumento y su comprensión.

A base de los datos obtenidos se determinó el valor del Alpha de Cronbach que indica que el índice de consistencia interna para el cuestionario global es elevado con un puntaje de 0,972.

\section{CONSIDERACIONES ÉTICAS}

Previo a la fase de recolección de datos se procedió a entregar un consentimiento informado a los padres y madres de los niños, donde se especificaban los objetivos de la investigación y la confidencialidad de los datos, de uso exclusivo de esta investigación, la voluntad de participar con un criterio de inclusión para la muestra.

\section{RESULTADOS}

El perfil poblacional incluyó padres en su mayoría entre 15 y 25 años, de sexo femenino, con un nivel de instrucción de bachillerato.

En cuanto a la frecuencia de juego la mayor parte de niños desarrollaban actividades lúdicas en un tiempo superior a una hora diariamente. $Y$ de ese tiempo el mayor porcentaje de pa- dres juegan menos de 15 minutos al día junto a sus hijos. Sin embargo, el $66 \%$ de la población considera que el tiempo de juego compartido es suficiente.

La valoración del juego se ha subdividido en cuatro funciones: física, cognitiva, socioemocional y del lenguaje. Existen diferencias significativas entre la percepción de los padres en cada área, 
mientras que la función física y socioemocional del juego es ampliamente valorada por los padres, las funciones cognitiva y del lenguaje son menospreciadas dentro del marco del desarrollo.

La función física es calificada en el $76 \%$ de la población como importante para el desarrollo infantil, lo que indica que la mayoría de los padres reconocen el papel del juego en el desarrollo del área motriz del niño, en referencia a la coordinación dinámica general, coordinación óculo-manual y equilibrio.

Percepción semejante es atribuida en la función socioemocional donde el 96\% de la población considera que el juego aporta al desarrollo de esta área, desglosado en la comprensión de reglas, acciones de colaboración, la expresión de emociones y la socialización.

Caso distinto ocurre en la función cognitiva donde el $68 \%$ de los padres no conciben al juego como un medio para la estimulación intelectiva de los niños, en indicadores como la imaginación, resolución de problemas, la discriminación y clasificación de objetos.

Dentro de la función del juego en el desarrollo del lenguaje, el $56 \%$ de padres no considera que tenga injerencia en la estimulación de esta área, en aspectos como la emisión de sonidos, el aumento de vocabulario y la asignación de signo al símbolo. Los resultados antes expuestos se detallan en la tabla 2.

Tabla 2. Cuadro resumen de valoración de las funciones del juego

\begin{tabular}{|l|c|c|c|c|}
\hline Valoración & Función Física & Función cognitiva & Función socioemocional & $\begin{array}{c}\text { Función en el } \\
\text { lenguaje }\end{array}$ \\
\hline Nada importante & $0 \%$ & $16 \%$ & $0 \%$ & $18 \%$ \\
\hline Poco importante & $24 \%$ & $52 \%$ & $4 \%$ & $38 \%$ \\
\hline Desvalorización & $24 \%$ & $68 \%$ & $4 \%$ & $56 \%$ \\
\hline Importante & $48 \%$ & $24 \%$ & $62 \%$ & $44 \%$ \\
\hline Muy importante & $28 \%$ & $8 \%$ & $34 \%$ & $0 \%$ \\
\hline Valorización & $76 \%$ & $32 \%$ & $96 \%$ & $44 \%$ \\
\hline
\end{tabular}

Elaboración: autoras

Paralelamente se estableció una frecuencia de juego compartido entre padres e hijos donde las áreas con una mejor percepción de su funcionalidad 
obtuvieron mayores puntajes. En el área física el $76 \%$ de padres practicaban junto a sus hijos habitualmente juegos que incluyeran actividades como saltar, trepar, correr o la manipulación de objetos. De igual forma, la frecuencia de juego, que estimula el área socioemocional en el $76 \%$, de la población es alta, entre los cuales se incluyen los juegos reglados, cooperativos o sociales.

En el lado inverso se encuentra la frecuencia de juego compartido en la función cognitiva, donde el $68 \%$ de la población pocas veces practican juegos que circunscribieran la creación de per- sonajes, historias o la aplicación de nociones comparativas. En tanto la frecuencia con que los niños realizan juegos que motivan el desarrollo del lenguaje junto a sus padres, en el $58 \%$ de la población fue baja.

En la percepción global, el 58\% de la población indica que el juego es una actividad que promueve el desarroIlo, en tanto el $42 \%$ restante la identifican con una actividad de distracción. Si bien nadie lo ubica como una pérdida de tiempo, no todos le asignan un papel dentro del desarrollo.

\section{DISCUSIÓN}

El juego se muestra como una actividad importante en el desarrollo vital de los niños. Por medio de este relacionan conocimientos que han adquirido, formando de este modo procesos de aprendizaje individual. En la primera etapa de su desarrollo, el juego, representa un aporte en el desarrollo cognitivo, social, emocional y en la formación de la personalidad. A través del juego los niños enriquecen su mente, estimulan su fantasía, crean situaciones y les dan solución.

Según Contreras (2014), en los primeros siete años el niño atraviesa un complicado camino de desarrollo. Esto se refleja claramente en los juegos y su complejidad en la resolución de tareas que por iniciativa propia los niños emprenden, la complejidad parte de la acción del juego solo o acompañado. En el desarrollo básico de los niños se evidencia la necesidad de explorar el mundo que les rodea ante esta tarea son los padres quienes han de ayudarle en su insaciable curiosidad y contestar a sus constantes cuestionamientos.

Los niños, aunque tengan compañeros de juegos reales, pueden albergar también uno o varios compañeros imaginarios. No será raro ver a los niños hablar en tonos distintos de voz y tener una larga y curiosa conversación consi- 
go mismo, está jugando, esta afirmación se obtiene en el estudio realizado por Recca (1986), quien puede describir con claridad la función del juego como una estrategia de creatividad y búsqueda de resolución en niños de 2 a 4 años, con su incipiente desarrollo neurocognitivo, los niños de estas edades buscan descubrir las regularidades relacionadas con la formación del pensamiento, imaginación, cualidades morales, hábitos colectivos y capacidades de creación.

Con estos antecedentes podemos focalizarnos en los resultados que se revelan en el presente estudio cuyo objetivo principal de la investigación fue determinar la valoración de la función del juego desde la visión de los padres. Identificando que la mayor parte en un $62 \%$, consideraron importante el papel global del juego en el desarrollo infantil; sin embargo más de un cuarto de la población (38\%) lo valoró como poco importante para el mismo. De lo que se desprende que el $42 \%$ de los padres consideró al juego como una actividad meramente de distracción.

Loredo (2005) confirma este dato afirmando que "aunque la mayoría de los padres consideran que los juegos y los juguetes son acciones y objetos destinados a mantener a los niños entreteni- dos y contentos, es conveniente insistir que también constituyen un método de adiestramiento y aprendizaje" (p. 214). En tanto para Payá (2007) el problema reside en que algunos padres todavía conservan una concepción del juego como una pérdida de tiempo y se indignan porque sus hijos en edad preescolar no hacen más que jugar en clase, en lugar de aprender algo más productivo.

En un análisis por cada dimensión del juego se evidenció que la función cognitiva es la que recibe menor relevancia y frecuencia desde la apreciación de los padres. Contrario a los aportes de las teorías del juego que enfatizan en el valor intelectual del mismo. Fergus menciona que "El juego facilita el aprendizaje, ya que expone al niño a nuevas experiencias y a nuevas posibilidades de enfrentar el mundo" (2006, pág. 30)

Uno de los autores dentro de este enfoque es Piaget (1961) quién parte de que el juego es una forma de asimilación, que consiste en adaptar los hechos de la realidad a esquemas ya existentes. Identifica en el juego a la vez la expresión y la condición del desarrollo del niño. Debido a que involucra la consolidación de las actividades físicas y mentales que se han aprendido, atribuyendo a cada etapa un tipo de juego. 


\section{CONCLUSIÓN}

Se observa que en el grupo estudiado, las familias le atribuyen escaso valor al juego como un factor del desarrollo en las funciones cognitiva y del lenguaje, por lo que es necesario que se establezca un plan de educación para las familias a fin de que ellas se conviertan en un elemento para un mejor desarrollo de estas funciones.

Al considerar que lo que ocurre en este Centro es una pauta cultural que se repite en otros centros según corroboran las investigaciones a nivel internacional y tomando en cuenta que el desarrollo alcanzado durante la etapa preescolar tiene incidencia a largo plazo en la vida del individuo, se destaca la importancia la formulación de políticas públicas orientadas para que las familias, los centros de cuidado y demás instituciones que atienden el desarrollo de los niños en la etapa preescolar trabajen alrededor de esta problemática. 


\section{REFERENCIAS}

Arango, M. (1997). Juguemos con los niños. Bogotá: Ediciones Gamma.

Bateson, G. (1972). Pasos hacia una ecología de la mente. Buenos Aires: Lohlé-Lumen.

Constitución de la República del Ecuador. (2008). Ciudad Alfaro: Asamblea Constituyente.

Deza, S. (2015). Factores de riesgo y protección en niños y adolescentes en situación de pobreza de instituciones educativas en Villa El Salvador. Av.psicol. vol 23, 231-240.

Feldman, R. (2008). Desarrollo en la infancia. México: Pearson Educación .

Fergus, P. (2006). El juego, su importancia en el desarrollo psicológico del niño y adolescente. México: Editorial Trillas.

Hernández, R. (2010). Metodología de Investigación. México: Mc GrawHill.

Hoffman, L., Paris, S., \& Hall, E. (1995). Psicología del desarrollo hoy. Madrid: McGraw-Hill.

Irwin, L. (2007). Primera infancia: un potente ecualizador. Recuperado el 15 de 09 de 2016, de http://www. who.int/social_determinants/publications/early_child_dev_ecdkn_es.pdf

Loredo, A. (2005). El juego y los juguetes: un derecho olvidado de los niños. Infancia sana, 214-219.
Minuchin, S. (1982). Familias y Terapia Familiar. Barcelona: Gedisa.

Muñoz. (2006). La familia como contexto del desarrollo infantil. Huelva: Universidad de Huelva.

Organización Mundial de la Salud. (2013). El desarrollo del niño en la primera infancia y la discapacidad. Recuperado el 14 de 09 de 2016, de http://unesdoc.unesco.org/images/0006/000695/06 9549so.pdf

Payá. (2007). La actividad lúdica en la historia de la educación española contemporánea. Valencia, Universidad de Valencia: Tesis doctoral.

Piaget. (1961). La formación del símbolo en el niño. Recuperado el 14 de 09 de 2016, de http://bloguamx. byethost10.com/ wp-content/ uploads/2015/04/formacic2a6n-del-simbolo-piaget.pd$f ? i=1$

Salles, C. (2011). Las competencias parentales en la familia contemporánea: descripción, promoción y evaluación. Educación social: revista de intervención socioeducativa, 30.

SENPLADES. (2013). Plan Nacional del Buen Vivir 2013-2017.

Von Foerster, H. (2000). Construyendo una realidad. En P. Watzlawick, La realidad inventada (págs. 17 - 38). Barcelona: Gedisa. 